OPEN ACCESS

Edited by:

Daniela De Biase,

Sapienza Università di Roma, Italy

Reviewed by:

Octavio Loera,

Universidad Autónoma Metropolitana,

Mexico

Juan Carlos Aon,

GlaxoSmithKline, United States

${ }^{*}$ Correspondence:

Yueqing Cao

yueqingcao@cqu.edu.cn

Yuxian Xia

yuxianxia@cqu.edu.cn

Specialty section:

This article was submitted to Microbial Physiology and Metabolism,

a section of the journal

Frontiers in Microbiology

Received: 07 June 2018

Accepted: 30 July 2018

Published: 21 August 2018

Citation:

Zhang E, Cao Y and Xia Y (2018) Ethanol Dehydrogenase I Contributes to Growth and Sporulation Under Low

Oxygen Condition via Detoxification

of Acetaldehyde in Metarhizium

acridum. Front. Microbiol. 9:1932.

doi: 10.3389/fmicb.2018.01932

\section{Ethanol Dehydrogenase I Contributes to Growth and Sporulation Under Low Oxygen Condition via Detoxification of Acetaldehyde in Metarhizium acridum}

\author{
Erhao Zhang ${ }^{1,2,3}$, Yueqing $\mathrm{Cao}^{1,2,3 *}$ and Yuxian Xia ${ }^{1,2,3 *}$ \\ ${ }^{1}$ School of Life Sciences, Chongqing University, Chongqing, China, ${ }^{2}$ Chongqing Engineering Research Center for Fungal \\ Insecticides, Chongqing, China, ${ }^{3}$ Key Laboratory of Gene Function and Regulation Technologies under Chongqing \\ Municipal Education Commission, Chongqing, China
}

The entomopathogenic fungi encounter hypoxic conditions in both nature and artificial culture. Alcohol dehydrogenases (ADHs) are a group of oxidoreductases that occur in many organisms. Here we demonstrate that an alcohol dehydrogenase I, MaADH1, in the locust-specific fungal pathogen, Metarhizium acridum, functions in acetaldehyde detoxification mechanism under hypoxic conditions in growth and sporulation. The MaADH1 was highly expressed in sporulation stage under hypoxic conditions. Compared with a wild-type strain, the $\triangle M a A D H 1$ mutant showed inhibited growth and sporulation under hypoxic conditions, but no impairment under normal conditions. Under hypoxic conditions, $\triangle \mathrm{MaADH1}$ mutant produced significant decreased alcohol, but significant increased acetaldehyde compared to wild type. M. acridum was sensitive to exogenous acetaldehyde, exhibiting an inhibited growth and sporulation with acetaldehyde added in the medium. MaADH1 did not affect virulence. Our results indicated that the MaADH1 was critical to growth and sporulation under hypoxic stress by detoxification of acetaldehyde in $M$. acridum.

Keywords: ethanol dehydrogenase, conidiation, growth, entomopathogenic fungi, hypoxic condition

\section{INTRODUCTION}

Entomopathogenic fungi are one of the widespread organisms belonging to different systematic groups. About 1000 species of these fungi have been recorded as insect pathogens (Fang et al., 2012). Entomopathogenic fungi are distributed throughout the world, from the plateaus to the plains, and from the deserts to the swamps (Zimmermann, 2007). Hence entomopathogenic fungi are exposed to a variety of environmental stresses, including hypoxia, heat, UV radiation, etc. (Ortiz-Urquiza and Keyhani, 2015). However, altitude has no impact on the presence of entomopathogenic fungi even in a range up to $5200 \mathrm{~m}$ where oxygen was only $50 \%$ of the sea level value (Peacock, 1998; Quesada-Moraga et al., 2007; Sun and Liu, 2008). Beauveria and Metarhizium had been reported in Nepal and Tibet, where the oxygen availability was only $70 \%$ of the normal 
atmospheric levels value (Peacock, 1998; Yubak Dhoj et al., 2008; Freed et al., 2011), showing their ability to adapt to a wide range of oxygen levels. Thus, entomopathogenic fungi can grow and produce conidia under hypoxic conditions in nature, and they likely have mechanisms to sense and respond to hypoxic stress.

Entomopathogenic fungi are one of the most promising agents for biological control of pest insects. Some species have been developed to biopesticide products. Up to 2016 September, 51 commercial fungal biopesticide products from 58 countries around the world are available, which are mostly based on the aerial conidia for at least 12 species of insect fungi (de Faria and Wraight, 2007; Muñiz-Paredes et al., 2017). As a microbial pesticide, M. acridum was used for locust and grasshopper control in Africa, Australia and Asia (Hunter et al., 2001; Lomer et al., 2001; Peng et al., 2008). Aerial conidia of entomopathogenic fungi produced in solid culture are of lipophilic nature, a superb property for developing an oil-based formulation suitable for ultra-low volume (ULV) application in arid environments (Bateman et al., 1993; Burges, 1998; Lomer et al., 2001). As aerobic organisms of entomopathogenic fungi, aeration is an important factor for aerial conidia production (MuñizParedes et al., 2017). However, the solid-state fermentation has some disadvantages, such as oxygen limitation due to poor diffusion of oxygen in solid substrate especially for mass production (Bartlett and Jaronski, 1998; Mienda et al., 2011). Therefore, aeration has a strong impact on the fungal growth and conidiation (Rahardjo et al., 2005). The increase of dissolved oxygen (DO) during sporulation in the liquid culture results in an increased blastospore production compared to normal condition (Issaly et al., 2005). In solid culture, conidia production was increased significantly under higher oxygen $\left(26 \% \mathrm{O}_{2}\right)$ compared to normal oxygen condition $\left(21 \% \mathrm{O}_{2}\right)$ (Tlecuitl-Beristain et al., 2010). Normally, entomopathogenic fungi encounter the challenges of hypoxic stress in nature or in artificial culture. However, up to now, the regulation mechanisms of hypoxic stress tolerance have not been explored in entomopathogenic fungi.

Alcohol dehydrogenases (ADHs) are a group of oxidoreductases that occur in many organisms facilitating the conversion between alcohols and aldehydes with the reduction of $\mathrm{NAD}+$. Alcohol dehydrogenases are widespread in the animals, plants, fungi and bacteria. However, the ADH type and number varied in different species. For example, there are five $\mathrm{ADH}$ members (ADH1, $\mathrm{ADH} 2, \mathrm{ADH} 3, \mathrm{ADH} 4$, and $\mathrm{ADH} 5)$, three in Saccharomyces cerevisiae (ADH1, $\mathrm{ADH} 2$, and $\mathrm{ADH} 3$ ) (de Smidt et al., 2008), two in Aspergillus nidulans (ADH1 and ADH3) (Reid and Fewson, 1994), and one in Mucor circinelloides (ADH1) (Rangel-Porras et al., 2005).

The functions of $\mathrm{ADH}$ members vary within the $\mathrm{ADH}$ family. In Thermoanaerobacter ethanolicus, three ADHs play different roles during ethanol formation (Zhou et al., 2017). In yeast, $\mathrm{ADH} 1$ is responsible for ethanol production from acetaldehyde and $\mathrm{NADH}$, contributing to fungal growth in the presence of alcohol (Plapp et al., 2013). Yeast ADH2 converts the ethanol accumulating to acetaldehyde under anaerobic conditions (de Smidt et al., 2008). In plant pathogenic fungus
Fusarium oxysporum, ADH1 disruption impaired growth under hypoxic conditions, diminished production of ethanol and affected the fungal disease development in tomato plants (Corrales Escobosa et al., 2011). In A. fumigatus, ADH could be induced by hypoxic condition, and influence fungal growth and pathogenesis (Grahl et al., 2011).

The $A D H$ genes widely exist in the insect pathogenic fungi of Metarhizium, Beauveria, and Cordyceps. Few past studies were reported on the function of alcohol dehydrogenase in entomopathogenic fungi. In $M$. anisopliae, $A D H 1$ is expressed during infection process and required for virulence (Callejas-Negrete et al., 2015). In this study, we explored the function of the $M a A D H 1$ gene by targeted disruption in $M$. acridum. Our results showed that $\triangle M a A D H 1$ had inhibited growth and sporulation under hypoxic conditions, while it had no impairment under normal oxygen conditions. When oxygen level was low, $\triangle M a A D H 1$ mutant produced decreased alcohol and increased acetaldehyde compared to wild type. Exogenous acetaldehyde had similar inhibition on sporulation as deletion of $M a A D H 1$ in $M$. acridum. These data suggested that $M a A D H 1$ was involved in hypoxic stress tolerance and contributed to conidiation under low oxygen condition.

\section{MATERIALS AND METHODS}

\section{Organisms and Culture Conditions}

Metarhizium acridum CQMa102 is wild type strain that was obtained from China General Microbiological Culture Collection Center (CGMCC, No. 0877). The fungal strains were propagated in $1 / 4$ SDAY medium, consisting of $1 \%$ dextrose, $0.25 \%$ mycological peptone, $0.5 \%$ yeast extract, and $2 \%$ agar (w/v). Escherichia coli DH5 $\alpha$ (DingGuo, Beijing, China) was used for routine cloning.

\section{Semi-Quantitative Reverse Transcription (qRT)-PCR and qRT-PCR}

Total RNAs were extracted from WT mycelia grown for 14 and $20 \mathrm{~h}$ days on plate, conidia (growing for 3 days), germinated conidia, appressoria formed on locust wing, and hyphal bodies grown for 5 days in hemolymph of infected locusts (Luo et al., 2013). Appressorium induction and hyphal body collection were performed as described previously (He and Xia, 2009). Total RNAs were extracted using TRIzol reagent (Invitrogen, United States). For first strand cDNA synthesis, $1 \mu \mathrm{g}$ total RNA was applied with an oligo-dT primer using the PrimeScript ${ }^{\mathrm{TM}}$ RT Master Mix (TaKaRa, Dalian, China). Semi-quantitative RT-PCR and qRT-PCR were performed using primers ADH1-EF and ADH1-ER. cDNA synthesis and qRTPCR were performed using the method described by Luo et al. (2013). All PCR amplifications were conducted in triplicate, the glyceraldehyde-3-phosphate dehydrogenase gene (gpd) was used as an internal control. Transcript ratios of target gene were evaluated using the $2^{-\Delta \Delta C T}$ method (Livak and Schmittgen, 2001). Primer sequences are listed in Supplementary Table S1. 


\section{MaADH1 Deletion, Complementation, and Over-Expression}

The MaADH1 gene was disrupted by homologous recombination. About $1 \mathrm{~kb}$ upstream and $1 \mathrm{~kb}$ downstream sequences of MaADH1 open reading frame (ORF) were amplified with primer pairs LF/LR and RF/RR from M. acridum genomic DNA. Amplification fragments were inserted into pK2-pB (Luo et al., 2013) separated by a selection marker Bar cassette. The recombinant plasmid was transformed into M. acridum with Agrobacterium tumefaciens according to Fang et al. (2004). Transformants were screened by PCR with primers $\mathrm{L} 1 / \mathrm{PR}$ and BF/R1 and confirmed by Southern blot. A plasmid for complementation was generated using primers $\mathrm{HF} / \mathrm{HR}$ to amplify the entire MaADH1 gene including $2 \mathrm{~kb}$ of the $M a A D H 1$ promoter, the coding sequence, and the terminator. The amplified fragment was inserted into pK2-EGFP-Sur containing the chorimuron ethyl resistance gene Sur (Luo et al., 2013). The pK2-Sur-ADH1-EGFP construct was transformed into the $\triangle M a A D H 1$. Transformants were screened on Czapekdox plates supplemented with $20 \mu \mathrm{g} / \mathrm{ml}$ chorimuron ethyl (Sigma, Bellefonte, PA, United States) and confirmed by DNA blots. Primers used for transformants construction are listed in Supplementary Table S1.

\section{Growth Under Different Oxygen Condition}

To examine the role of MaADH1 on fungal growth under different DO condition, WT, $\triangle M a A D H 1$, and complemented strain (CP) were grown in liquid culture under normal dissolvedoxygen and low DO condition, respectively. Conidia were inoculated into $1 / 4$ SDY liquid medium with a concentration of $1 \times 10^{5}$ conidia/ml in $50 \mathrm{ml}$ centrifuge tubes. Centrifuge tubes were sealed with ventilated films for normal oxygen condition. To get lower DO, $20 \mu \mathrm{l}$ liquid $\mathrm{CO}_{2}$ was added in the culture mixture to obtain about $18 \%$ oxygen concentration at the initial time of growth ( $80 \%$ of normal oxygen level), then the tubes were sealed with caps and shaken at $250 \mathrm{rpm}$ at $28^{\circ} \mathrm{C}$. The fungal cultures were collected by centrifugation every $12 \mathrm{~h}$ during a 5 -day growing period. The pellets were weighed and compared among fungal strains. The transcription of $\mathrm{MaADH1}$ and $\mathrm{ADHs}$ enzyme activity were also determined in these samples. Total RNAs were extracted from these fungal cultures for transcription level analysis of MaADH1 by qRT-PCR as described above. The DO in liquid culture was detected with DO electrode (Mettler Toledo, Inpro6000, Switzerland).

\section{ADH Activity Assay}

The ADH activity assays were performed in reaction mixtures containing $25 \mu \mathrm{l}$ cell-free fractions, $1.0 \mathrm{ml}$ semicarbazide buffer ( $\mathrm{pH}$ 8.7), $1.5 \mathrm{mM} \mathrm{NAD}+$ solutions and $10 \mathrm{mM}$ ethanol. The reduction of NAD+ was monitored by the increased absorbance at $340 \mathrm{~nm}$. One unit of enzyme activity was defined as the amount of enzyme catalyzing the reduction of $1 \mu \mathrm{mol} \mathrm{NAD}+$ per min at $25^{\circ} \mathrm{C}$. Specific activity was expressed as units (U) per mg of protein. Protein concentration was determined using the BCA protein assay kit (Beyotime, China)

\section{Sporulation Determination}

Sporulation under hypoxic condition was determined in liquid medium and on solid rice grain. Fungal strains were grown in liquid medium under hypoxic condition as described above. After growing for 3 days, the fungal culture was collected and filtered with four layers of lens tissue to remove mycelia. Blastospore concentration in filtered solution was determined by hemocytometer under microscope. Two-stage technique was applied in solid-state fermentation, which involves submerged liquid fermentation and solid substrate fermentation. Rice grains were soaked in water for $5 \mathrm{~h}$, and then were autoclaved after removing water. Three-day fungal culture grown in liquid $1 / 4$ SDY medium $\left(1 \times 10^{6}\right.$ spore $\left./ \mathrm{ml}\right)$ under normal condition was mixed thoroughly with grains to a final concentration of $10 \%(\mathrm{~V} / \mathrm{W})$. The mixture was then filled into sealed glass tubes (diameter: $3.5 \mathrm{~cm}$, height: $11 \mathrm{~cm}$ ), and then fermented at $28^{\circ} \mathrm{C}$ for 14 days to produce conidia. The rice grains were shaken once every 2 days to prevent the grains sticking together. Fermented rice $(0.25 \mathrm{~g})$ was soaked in $1 \mathrm{ml} \mathrm{H}_{2} \mathrm{O}$, and vortexed to wash off the conidia from rice grains. Conidia number was determined by hemocytometer under microscope.

\section{Alcohol and Acetaldehyde Determination}

Alcohol and acetaldehyde determination was performed as described previously with minor modifications (Bernt and Gutmann, 1974; Rotariu et al., 2004). Fungal growth in liquid $1 / 4$ SDY under hypoxic condition were as described above. The cultures were collected every $12 \mathrm{~h}$ during a 5 -day growing period and then centrifuged to collect the supernatant. For alcohol determination, supernatant of $25 \mu \mathrm{l}$ was mixed thoroughly with $1.0 \mathrm{ml}$ semicarbazide buffer $(\mathrm{pH}$ 8.7) and $25 \mu \mathrm{lNAD+}$ solutions (Solarbio, Beijing, China). Alcohol dehydrogenase solution $(5 \mu \mathrm{l}$ ) sourced from S. cerevisiae (Solarbio, Beijing, China) was added to the mixture and then incubated at $25^{\circ} \mathrm{C}$ for $30 \mathrm{~min}$. The absorbance of the reaction mixture was read at $340 \mathrm{~nm}$. Standard curve was generated using $0-0.04 \%$ (v/v) ethanol solutions.

For acetaldehyde determination, $25 \mu \mathrm{l}$ of sample solution was added to a tube containing $300 \mu \mathrm{l}$ Tris- $\mathrm{HCl}$ buffer $(1 \mathrm{M}, \mathrm{pH}$ 8.0) and $25 \mu \mathrm{lNAD}+$ solutions $(1 \mathrm{mM})$. Acetaldehyde dehydrogenase (E.C 1.2.1.5) $(5 \mu \mathrm{l})$ was added to the mixture and then incubated at $25^{\circ} \mathrm{C}$ for $30 \mathrm{~min}$. The absorbance of the reaction mixture was read at $340 \mathrm{~nm}$. Standard curve was generated using $0-0.04 \%$ $(\mathrm{v} / \mathrm{v})$ acetaldehyde solutions prepared with phosphate buffer $\mathrm{pH}$ 9.0 containing $0.1 \mathrm{M} \mathrm{KCl}$.

\section{Effect of Acetaldehyde on Fungal Growth}

To investigate the effects of acetaldehyde on fungal growth, wild type strain was grown in liquid or solid medium containing 0.01 or $0.1 \%$ acetaldehyde. For liquid culture, conidia were inoculated in $1 / 4$ SDY in a $50 \mathrm{ml}$ centrifuge tube at a final concentration of $1 \times 10^{5}$ conidia $/ \mathrm{ml}$, and then shaken at $28^{\circ} \mathrm{C}$ with $250 \mathrm{rpm}$ for 4 days. Blastospore production was determined daily. For culture on plate, conidia $\left(1 \times 10^{6}\right.$ conidia $\left./ \mathrm{ml}\right)$ were spotted or spread on $1 / 4$ SDAY plate and grown for 14 days. Conidia production 
was determined every 3 days as described previously (Liu et al., 2010). The phenotype of fungal colonies was shown on 6th day of growth.

\section{Germination, Stress Tolerance, and Bioassay}

Germination rate was determined on normal medium (1/4 SDAY) and host insect wing [Locusta migratoria manilensis (Meyen)] as described previously (Liu et al., 2010).

Tolerance to heat and ultraviolet radiation was determined according to Liu et al. (2010). IT $_{50}$ (time for 50\% inhibition in germination rate by heat or UV irradiation) was compared among WT, $\triangle M a A D H 1$, and the CP.

Bioassay was performed using fifth instar nymphs of L. migratoria as described previously (Cao et al., 2012). Conidia suspensions $(5 \mu \mathrm{l})$ were topically inoculated on pronotum $\left(1 \times 10^{7}\right.$ conidia/ml liquid paraffin oil). Half-lethal time $\left(\mathrm{LT}_{50}\right)$ was calculated and compared between WT and $\triangle M a A D H 1$.

\section{Data Analysis}

Conidiation production, enzyme activity, ethanol, and acetaldehyde concentration, germination rate, $\mathrm{IT}_{50}$ and $\mathrm{LT}_{50}$, were statistically analyzed using one-way analysis of variance model (SPSS 16.0; SPSS Inc., Chicago, IL, United States). Tukey's honestly significant difference test was used to separate means at $p=0.05$.

\section{RESULTS}

\section{Gene Cloning and Molecular Characterization}

The full length of cDNA of MaADH1 is 1062 bp (Accession number KX021843). It encodes a predicted protein of 353 amino acids residues, with a deduced molecular weight of $37 \mathrm{KDa}$ and a $\mathrm{pI}$ of 7.61. The SignalP 3.0 program revealed no signal sequence, suggesting that MaADH1 is a cell-bound protein. MaADH1 contains typical ethanol dehydrogenase structural domains including NAD+ binding domain, $\mathrm{Zn}^{2+}$ binding site and ADH1 activity domain (Supplementary Figure S1A). Phylogenic analysis indicated that MaADH1 protein was closely related to ADH1 from the entomopathogenic fungi $M$. anisopliae (identity 94\%) and Beauveria bassiana (identity 73\%) (Supplementary Figure S1B).

\section{MaADH1 Is Induced in Hypoxic Condition}

The concentration of DO in culture fluctuated around the value of $21 \%$ under normal condition during growth period (Figure 1A). Under low oxygen condition, DO in culture was about $18 \%$ at the initial time and then gradually decreased along with the fungal growth. However, DO in $\triangle M a A D H 1$ culture was slightly higher than WT and CP strains (Figure 1A). In 1/4 SDY liquid medium, the $M a A D H 1$ had significantly increased transcription under low DO condition, with up to 10 times higher as compared to normal oxygen condition (Figure 1B). Transcription of MaADH1 gene was drastically increased after
2 days of growth under hypoxic condition, while normal oxygen condition had slight effect on MaADH1 transcription in this period (Figure 1B). Blastospores were produced substantially in liquid media during this period. These results demonstrated that $M a A D H 1$ might have a function in sporulation, especially sporulation under hypoxic condition.

Alcohol dehydrogenases enzyme activities were also determined in M. acridum under low and normal DO conditions. $\mathrm{ADH}$ activity showed a similar trend as the transcription results of MaADH1 (Figure 1C). The specific activity of ADHs was significantly increased under low DO condition compared to aerobic condition. The ADH enzyme activity increased from 1st day and reached to peak value of $40.5 \pm 1.5 \mathrm{U} / \mathrm{mg}$ after growing for 2.5 days under hypoxic condition, almost 2 times higher than that under aerobic condition $(21.5 \pm 0.9 \mathrm{U} / \mathrm{mg})$. A drastic decrease in ADHs activity was found at 3.5 days under aerobic condition, showing about $50 \%$ decrease compared to the peak value, while a $20 \%$ decrease was found at 3.5 day under hypoxic condition (Figure 1C). However, compared to transcription results of $M a A D H 1, \mathrm{ADH}$ enzyme activity did not have such drastic difference between normal and hypoxic condition (10 times versus 2 times increase) (Figure 1C). We supposed that this was due to the total $\mathrm{ADH}$ activity we determined in this study, and some other $\mathrm{ADH}$ members might require different biochemical conditions to measure the activity or have relative higher activity under normal oxygen condition, thus obscuring the difference in total $\mathrm{ADH}$ activity under normal and hypoxic condition.

\section{MaADH1 Is Highly Expressed in Sporulation}

The expression of MaADH1 at different stages of the life cycle and under low DO condition in liquid culture was analyzed by semi-quantitative RT-PCR and qRT-PCR. On 1/4 SDAY plate, the $M a A D H 1$ was highly transcribed in sporulation stage (3-day culture on plate) and poorly transcribed in mycelium, appressorium and hyphal body formation stages (Figures 2A,B). Consistent with the transcription results, MaADH1-EGFP fusion expression showed that the fungus had strong green fluorescence in sporulation stage on the $3 \mathrm{rd}$ day grown on plate, while weak or no signal was found in mycelia, germ tubes, appressorium and hyphal body (Figure 2C).

\section{MaADH1 Contributes Fungal Growth and Conidiation Under Low Oxygen Condition}

Targeted gene disruption of $M a A D H 1$ was generated by homologous recombination (Supplementary Figure S2A). Correct integration events in transformants of the deletion and subsequent complementation mutants were confirmed by Southern blotting (Supplementary Figure S2B). To examine the role of $M a A D H 1$ in fungal growth, biomass of fungal strains in liquid culture was analyzed under normal dissolved-oxygen and low DO condition, respectively. Results showed that biomass of $\triangle M a A D H 1$ had no significant difference compared with WT and $\mathrm{CP}$ under normal oxygen condition (Figure 3A), while decreased 

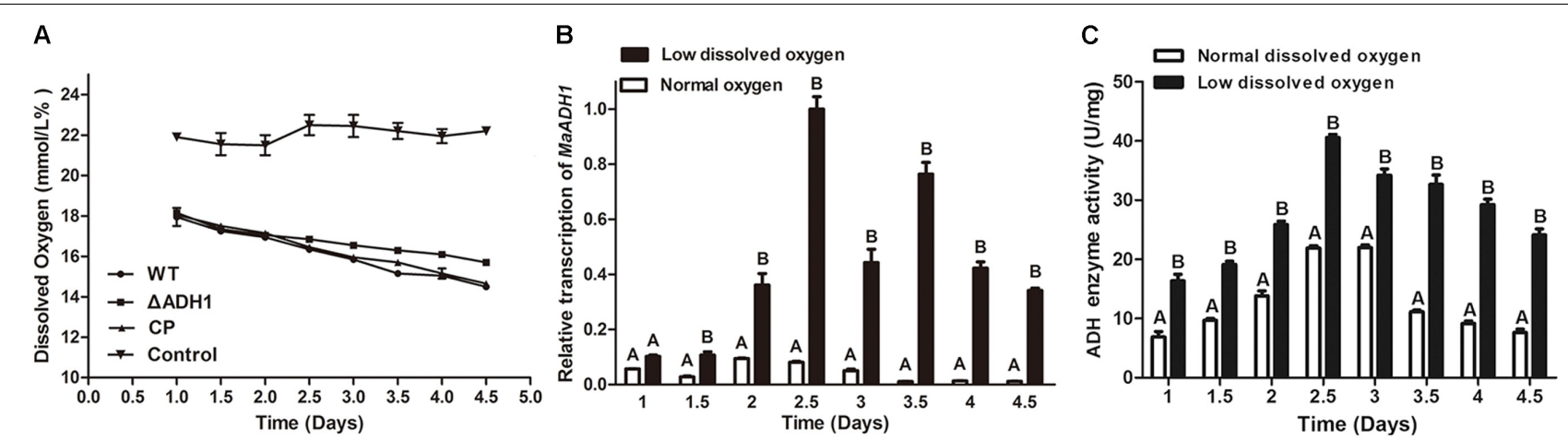

FIGURE 1 | Transcription and enzyme activity. (A) Dissolved oxygen under normal and low oxygen condition. (B) Relative expression of MaADH1 under hypoxic condition and normal condition. (C) ADH activity at different growth time under hypoxic condition and normal condition. A, B indicate significant difference at $p<0.01$.

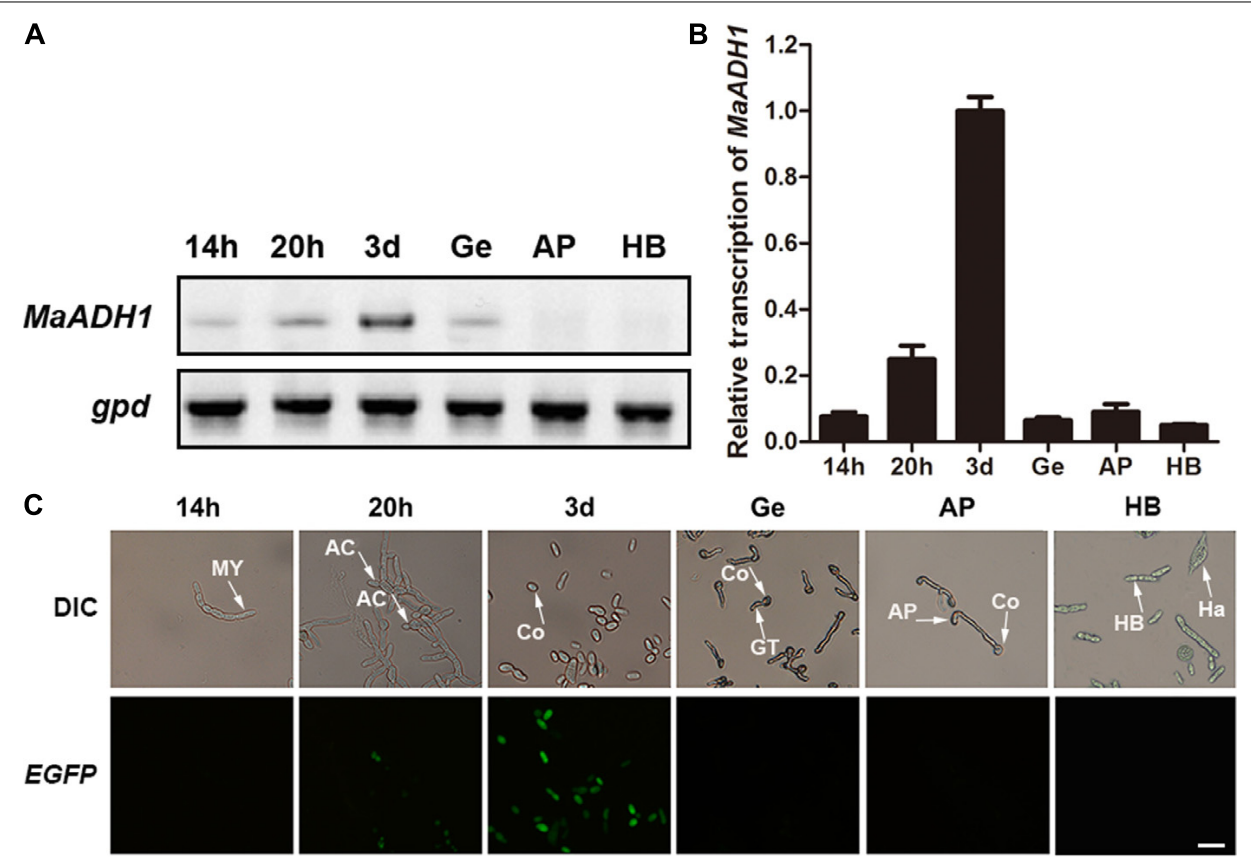

FIGURE 2 | Expression pattern of MaADH1 by semi-quantitative RT-PCR (A) and qRT-PCR (B) analysis of transcription of the MaADH1 gene on different life stages. (C) Fluorescence of MaADH1-EGFP in different stage. Ge, germination; AP, appressorium; HB, hyphal body; MY, Mycelium; AC, Arthroconidia; Co, conidia; GT, germ tube; Ha, hemocyte. Scale bar $=10 \mu \mathrm{m}$.

significantly after growing for 3 days under low oxygen condition (Figures $3 \mathbf{B}, \mathbf{C})$. The pellet of $\triangle M a A D H 1$ fungal culture was obviously less than WT and CP strains (Figure 3D). These results indicated that $M a A D H 1$ affected growth of M. acridum in liquid medium under low DO condition.

Conidiation of fungal strains on rice grains under low oxygen condition showed that rice grains in both WT and CP strains groups were covered with dark green conidia, while grains of $\triangle M a A D H 1$ group were covered with light-green conidia (Figure 4A). Microscopic analysis revealed that more white mycelia were found on rice surface in $\triangle M a A D H 1$ group compared to WT (Figure 4A). Quantitative conidiation analysis showed that $\triangle M a A D H 1$ had significantly decreased conidia production, almost $50 \%$ less than wild type strain, suggesting that $\mathrm{MaADH} 1$ also contributed to aerial conidiation in M. acridum under hypoxic condition (Figure 4B). Sporulation in liquid culture exhibited the same decreased trend as on rice grains in $\triangle M a A D H 1$ under hypoxic condition (Figures 4C,D).

MaADH1 did not affect conidia germination, heat and UV stress tolerance, and virulence (data not shown).

\section{MaADH1 Regulates Acetaldehyde Metabolism in M. acridum}

Alcohol dehydrogenases constitute a large family of enzymes responsible for the reversible oxidation of alcohols to aldehydes with the concomitant reduction of NAD + or NADP + . To 

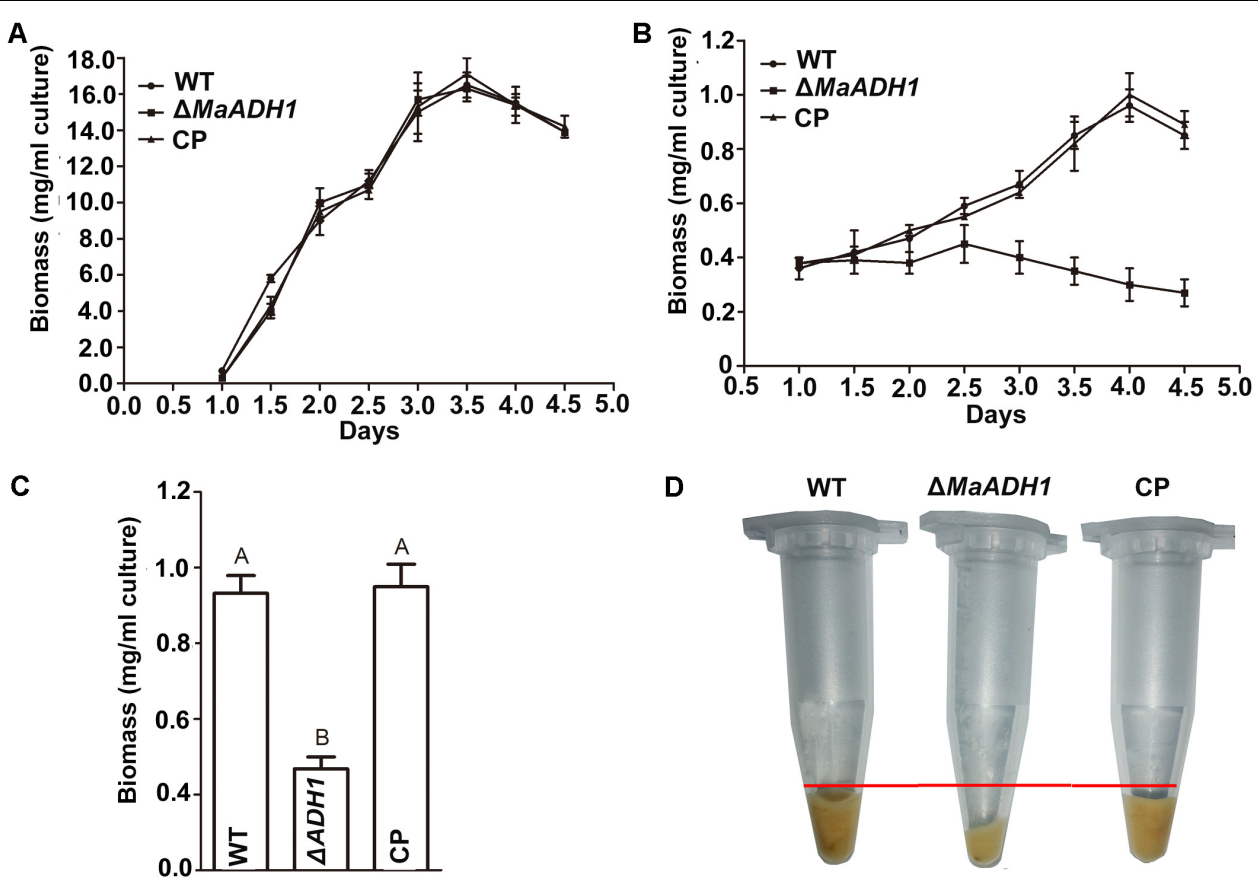

FIGURE 3 | Biomass analysis. (A) Time course study of dry weight under normal oxygen condition. (B) Biomass under low dissolved-oxygen condition. Dry weight (C) and pellets (D) on 3rd day under low dissolved-oxygen condition. Error bars are standard deviations of three trials. A, B indicate significant difference at $p<0.01$.

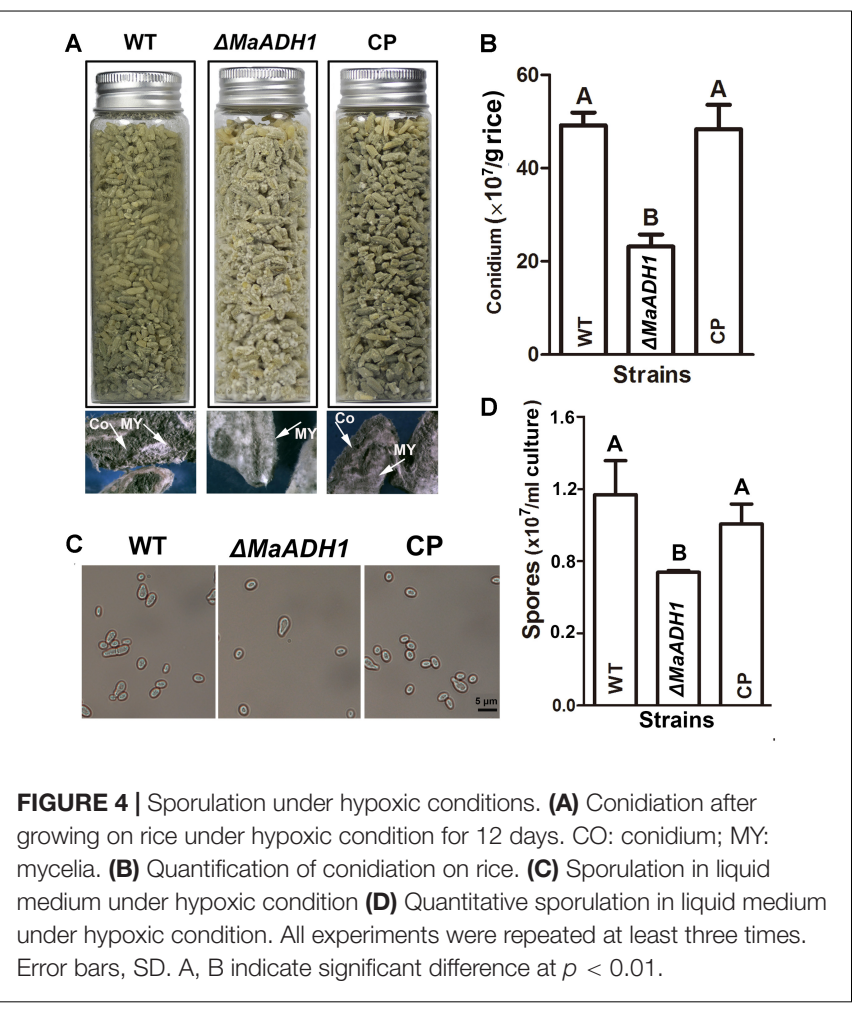

further explore the roles of MaADH1 under low oxygen condition, the ethanol and acetaldehyde in the liquid culture were measured. Compared to wild type, the $\triangle M a A D H 1$ culture had significant decreased alcohol concentration (about $0.005 \%$ in $\triangle M a A D H 1$ versus to $0.008 \%$ in WT) (Figure $\mathbf{5 A}$ ) and significant increased concentration of acetaldehyde (about $0.01 \%$ in $\triangle M a A D H 1$ versus to $0.002 \%$ in WT) (Figure 5B). Considering the inhibited growth results of $\triangle M a A D H 1$, it could be speculated that acetaldehyde accumulation in the culture was toxic to M. acridum.

\section{Acetaldehyde Affected Conidiation in M. acridum}

To further analysis the influence of acetaldehyde on growth and sporulation, wild type strain was inoculated on 1/4 SDAY plate and in liquid media containing acetaldehyde $(0.01$ or $0.1 \%$, $\mathrm{v} / \mathrm{v})$. Results showed that acetaldehyde inhibited the growth of $M$. acridum at concentrations of both 0.1 and $0.01 \%$ on plate (Figure 6A) or in liquid medium (Figure 6B), suggesting the acetaldehyde accumulation caused by $M a A D H 1$ deletion was sufficient to inhibit fungal growth. Compared to control, sporulation production was decreased significantly both in liquid (Figure 6C) and on solid medium (Figure 6D) containing acetaldehyde, showing about $50 \%$ decrease during the inspection period. These results suggested that acetaldehyde had negative effect on sporulation in M. acridum.

\section{DISSCUSSION}

In this study, we identified an $A D H 1$ gene from the entomopathogenic fungus $M$. acridum, also explored its function in sporulation and growth under low or normal 

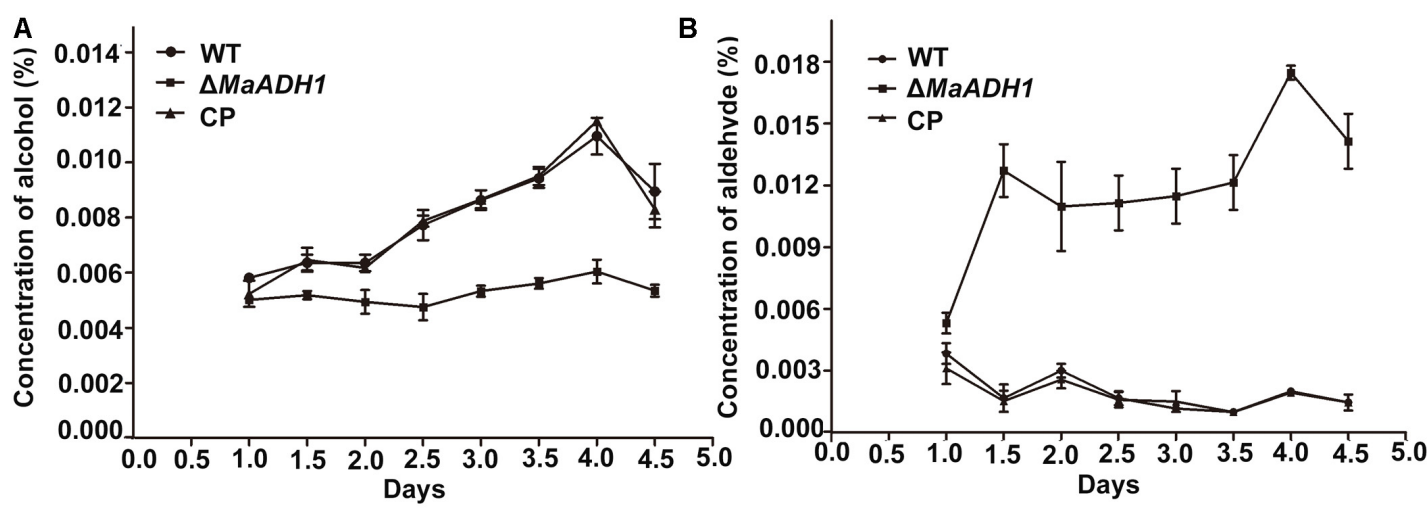

FIGURE 5 | Alcohol and acetaldehyde production under low oxygen condition. Concentration of alcohol (A) and acetaldehyde (B) in liquid medium at the different cultural time. Error bars are standard deviations of three trials.

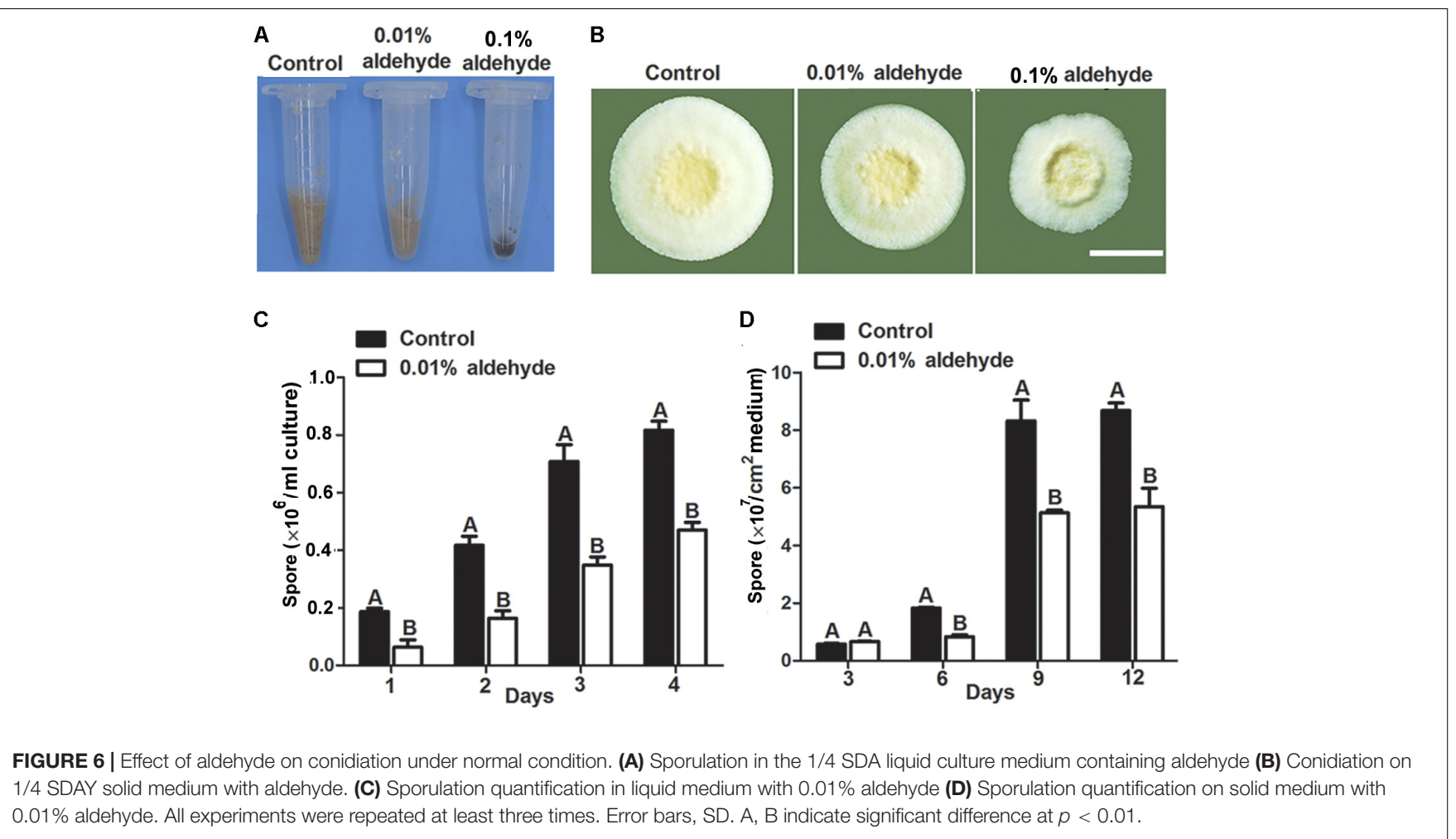

oxygen condition. Our results showed that the MaADH1 gene contributed to growth and sporulation under low oxygen condition via detoxification of acetaldehyde. However, this gene was not involved in virulence and UV/heat stress tolerance in M. acridum.

MaADH1 mainly accounted for conversion from acetaldehyde to ethanol under hypoxic condition in M. acridum. Similar as reported in some other fungi (Leskovac et al., 2002; Rangel-Porras et al., 2005), The MaADH1 had an up-regulated transcription and increased enzyme activity under low oxygen condition, suggesting its role under micro-oxygen conditions. Our data revealed a decrease in ethanol production and an increase in acetaldehyde production in $\triangle M a A D H 1$ culture, indicating a block in ethanol production from acetaldehyde under hypoxic condition when the $M a A D H 1$ gene was impaired. This physiological function was consistent with earlier reports in some fungi, such as S. cerevisiae (Leskovac et al., 2002; de Smidt et al., 2008), Candida maltose (Lin et al., 2010), F. oxysporum (Corrales Escobosa et al., 2011), M. anisopliae (Callejas-Negrete et al., 2015), and M. circinelloides (Rangel-Porras et al., 2005).

$\mathrm{ADH} 1$ is an enzyme strategy to detoxify acetaldehyde under low oxygen condition in M. acridum. Acetaldehyde is an inhibitor of a wide range of metabolic activities and is toxic to fungi (Aranda and del Olmo, 2004). Acetaldehyde at high concentrations can even stop yeast cell growth (Stanley et al., 1993). Consistence to these reports, our results showed 
that acetaldehyde had strong adverse effect on growth in M. acridum (Figure 5). ADH1 is a fermentative enzyme to catalyze the transformation of acetaldehyde to ethanol, reducing the accumulation of acetaldehyde in cells. In our study, deletion of the $M a A D H 1$ gene resulted in accumulation of acetaldehyde (reach to a concentration of $0.01 \%$ ) in culture (Figure 5) and inhibited growth and sporulation. The inhibition effect was in accordance with that of exogenous acetaldehyde (at a concentration of $0.01 \%$ ) on the growth of wild type strain (Figure 6). It is therefore reasonably supposed that MaADH1 played a major role in overcoming acetaldehyde toxicity in the liquid or solid-state fermentation in M. acridum.

The ability to sense and respond to changes in oxygen is essential for the survival of prokaryotic and eukaryotic organisms (Giaccia et al., 2004). Our data showed that fungal blastospore production in liquid culture appeared to be clearly related to the DO concentration. Sporulation was significantly decreased with low DO concentration (Figure 3). These results were in accordance with the previous report in M. flavoviride (Issaly et al., 2005). Ethanol could be detected when oxygen was depleted in part of the solid medium (Oostra et al., 2001), which also agreed to our data (Figure 5). Therefore, microbes would develop a strategy to decrease the adverse effect of hypoxic condition. As a group of fermentative enzymes, ADHs might play a role in this process. The physiological functions vary among different $\mathrm{ADH}$ members. In Thermoanaerobacter ethanolicus, AdhE primarily functions in acetaldehyde production, and AdhB has high activity for ethanol production, but does not contribute to acetaldehyde production (Zhou et al., 2017). In yeast, $\mathrm{ADH} 1$ and $\mathrm{ADH} 2$ are responsible for the inverse conversion between ethanol and acetaldehyde (de Smidt et al., 2008). Therefore, ADHs members might coordinate to overcome the hypoxic stress. Our data revealed that $M a A D H 1$ was induced in conidiation process under low oxygen condition, showing a much higher transcription compared to normal oxygen condition (Figure 2). This has also been reported in other $\mathrm{ADH}$ members in $A$. fumigatus (Grahl et al., 2011). Oxygen limitation has been the major concern in fungal solid-state fermentation (Bartlett and Jaronski, 1998; Mienda et al., 2011). Disruption of MaADH1 resulted in decreased conidiation on rice grain (Figure 4), suggesting a role of $\mathrm{MaADH} 1$ under hypoxic condition. Better understanding the regulation mechanisms and comprehensive utilization of $\mathrm{ADH}$ members in engineered strain construction could improve the fungal fermentation, especially in high density fermentation.

$A D H 1$ did not contribute to virulence in M. acridum. The infection process of entomopathogenic fungi involves attachment of conidia to the insect cuticle, germination of conidia, development of appressorium, formation of the penetration

\section{REFERENCES}

Aranda, A., and del Olmo, M. L. (2004). Exposure of Saccharomyces cerevisiae to acetaldehyde induces sulfur amino acid metabolism and polyamine transporter genes, which depend on Met4p and Haalp transcription factors, respectively. Appl. Environ. Microbiol. 70, 1913-1922. doi: 10.1128/AEM.70.4.1913-1922. 2004 peg to penetrate the insects' cuticle and killing the host insect by the growth of invasive hyphae in hemolymph and toxin produced by the fungi (Clarkson and Charnley, 1996). Our results revealed that $\mathrm{ADH} 1$ had very low transcription during germination and almost no transcription in appressoria and hyphal bodies. Accordingly, no fluorescence was detected in hyphal bodies and appressoria for ADH1-EGFP fused expression in $M$. acridum, suggesting ADH1 did not involve in pathogenicity process. Bioassay results against locusts were in accordance with the transcription profiles of $M a A D H 1$, showing no contribution of $\mathrm{ADH} 1$ to virulence (data now shown). However, our results were not consistent with the findings in $M$. anisopliae, which $\mathrm{ADH} 1$ is expressed during insect colonization and required for full virulence (Callejas-Negrete et al., 2015). ADH1 is also involved in virulence in plant pathogenic fungus $F$. oxysporum (Corrales Escobosa et al., 2011) and human pathogenic fungus C. albicans (Ku et al., 2017) and A. fumigatus (Grahl et al., 2011). This variation in $\mathrm{ADH} 1$ function illustrates that $\mathrm{ADH} 1$ might have distinct roles for a pathogenic fungi during interactions with host organisms.

In summary, our results demonstrated that the function of MaADH1 was to convert acetaldehyde to alcohol and eliminate side effect of acetaldehyde on fungal growth and sporulation under hypoxic conditions. Further better understanding the function and regulation of the $M a A D H 1$ may potentially help to improve high density fermentation in entomopathogenic fungi.

\section{AUTHOR CONTRIBUTIONS}

EZ conducted the main experiment and wrote the manuscript. YX and YC conceived and designed the experiments. EZ and YC analyzed the data. YX and YC provided technical oversight and critical manuscript review and editing.

\section{FUNDING}

This work was supported by the National Science and Technology Major Project of China (2017YFD0201208) and National Science Foundation of China (31272090).

\section{SUPPLEMENTARY MATERIAL}

The Supplementary Material for this article can be found online at: https://www.frontiersin.org/articles/10.3389/fmicb. 2018.01932/full\#supplementary-material

Bartlett, M. C., and Jaronski, S. T. (1998). "Mass production of entomogenous fungi for biological Control of insects," in Fungi in Biological Control Systems, ed. M. N. Burge, 61-85. Manchester: Manchester University Press.

Bateman, R. P., Carey, M., Moore, D., and Prior, C. (1993). The enhanced infectivity of Metarhizium flavoviride in oil formulations to desert locusts at low humidities. Ann. Appl. Biol. 122, 145-152. doi: 10.1111/j.1744-7348.1993. tb04022.x 
Bernt, E., and Gutmann, I. (1974). "Ethanol determination with alcohol dehydrogenase and NAD," in Methods of Enzymatic Analysis, Vol. 3, ed. H. A. Bergmeyer (Weinheim: Verlag Chemie), 1499-1502.

Burges, H. D. (1998). "Formulation of mycoinsecticides," in Formulation of Microbial Biopesticides: Beneficial Microorganisms, Nematodes and Seed Treatments, ed. H. D. Burges (Dordrecht: Kluwer Academic Publisher), 132185. doi: 10.1007/978-94-011-4926-6

Callejas-Negrete, O. A., Torres-Guzman, J. C., Padilla-Guerrero, I. E., EsquivelNaranjo, U., Padilla-Ballesteros, M. F., Garcia-Tapia, A., et al. (2015). The Adh1 gene of the fungus Metarhizium anisopliae is expressed during insect colonization and required for full virulence. Microbiol. Res. 172, 57-67. doi: 10.1016/j.micres.2014.11.006

Cao, Y., Zhu, X., Jiao, R., and Xia, Y. (2012). The Magas1 gene is involved in pathogenesis by affecting penetration in Metarhizium acridum. J. Microbiol. Biotechnol. 22, 889-893. doi: 10.4014/jmb.1111.11055

Clarkson, J. M., and Charnley, A. K. (1996). New insights into the mechanisms of fungal pathogenesis in insects. Trends Microbiol. 4, 197-203. doi: 10.1016/0966$842 \mathrm{X}(96) 10022-6$

Corrales Escobosa, A. R., Rangel Porras, R. A., Meza Carmen, V., Gonzalez Hernandez, G. A., Torres Guzman, J. C., Wrobel, K., et al. (2011). Fusarium oxysporum Adh1 has dual fermentative and oxidative functions and is involved in fungal virulence in tomato plants. Fungal Genet. Biol. 48, 886-895. doi: 10.1016/j.fgb.2011.06.004

de Faria, M., and Wraight, S. P. (2007). Mycoinsecticides and mycoacaricides: a comprehensive list with worldwide coverage and international classification of formulation types. Biol. Control. 43, 237-256. doi: 10.1016/j.biocontrol.2007. 08.001

de Smidt, O., du Preez, J. C., and Albertyn, J. (2008). The alcohol dehydrogenases of Saccharomyces cerevisiae: a comprehensive review. FEMS Yeast Res. 8, 967-978. doi: 10.1111/j.1567-1364.2008.00387.x

Fang, W., Azimzadeh, P., and St Leger, R. J. (2012). Strain improvement of fungal insecticides for controlling insect pests and vector-borne diseases. Curr. Opin. Microbiol. 15, 232-238. doi: 10.1016/j.mib.2011. 12.012

Fang, W., Zhang, Y., Yang, X., Zheng, X., Duan, H., Li, Y., et al. (2004). Agrobacterium tumefaciens-mediated transformation of Beauveria bassiana using an herbicide resistance gene as a selection marker. J. Invertebr. Pathol. 85, 18-24. doi: 10.1016/j.jip.2003.12.003

Freed, S., Jin, F. L., and Ren, S. X. (2011). Determination of genetic variability among the isolates of Metarhizium anisopliae var. anisopliae from different geographical origins. World J. Microbiol. Biot. 27, 359-370. doi: 10.1007/ s11274-010-0466-8

Giaccia, A. J., Simon, M. C., and Johnson, R. (2004). The biology of hypoxia: the role of oxygen sensing in development, normal function, and disease. Genes Dev. 18, 2183-2194. doi: 10.1101/gad.1243304

Grahl, N., Puttikamonkul, S., Macdonald, J. M., Gamcsik, M. P., Ngo, L. Y., Hohl, T. M., et al. (2011). In vivo hypoxia and a fungal alcohol dehydrogenase influence the pathogenesis of invasive pulmonary Aspergillosis. PLoS Pathog. 7:e1002145. doi: 10.1371/journal.ppat.1002145

He, M., and Xia, Y. (2009). Construction and analysis of a normalized cDNA library from Metarhizium anisopliae var. acridum germinating and differentiating on Locusta migratoria wings. FEMS Microbiol. Lett. 291, 127-135. doi: 10.1111/j. 1574-6968.2008.01447.x

Hunter, D. M., Milner, R. J., and Spurgin, P. A. (2001). Aerial treatment of the Australian plague locust, Chortoicetes terminifera (Orthoptera:Acrididae) with Metarhizium anisopliae (Deuteromycotina: Hyphomycetes). Bull. Entomol. Res. 91, 93-99.

Issaly, N., Chauveau, H., Aglevor, F., Fargues, J., and Durand, A. (2005). Influence of nutrient, $\mathrm{pH}$ and dissolved oxygen on the production of Metarhizium flavoviride Mf189 blastospores in submerged batch culture. Process Biochem. 40, 1425-1431. doi: 10.1016/j.procbio.2004. 06.029

Ku, M., Baek, Y. U., Kwak, M. K., and Kang, S. O. (2017). Candida albicans glutathione reductase downregulates Efg1-mediated cyclic AMP/protein kinase A pathway and leads to defective hyphal growth and virulence upon decreased cellular methylglyoxal content accompanied by activating alcohol dehydrogenase and glycolytic enzymes. Biochim. Biophys. Acta 1861, 772-788. doi: 10.1016/j.bbagen.2016.10.010
Leskovac, V., Trivić, S., and Peričin, D. (2002). The three zinc-containing alcohol dehydrogenases from baker's yeast, Saccharomyces cerevisiae. FEMS Yeast Res. 2, 481-494. doi: 10.1111/j.1567-1364.2002.tb00116.x

Lin, Y., He, P., Wang, Q., Lu, D., Li, Z., Wu, C., et al. (2010). The alcohol dehydrogenase system in the xylose-fermenting yeast Candida maltosa. PLoS One 5:e11752. doi: 10.1371/journal.pone.0011752

Liu, J., Cao, Y., and Xia, Y. (2010). Mmc, a gene involved in microcycle conidiation of the entomopathogenic fungus Metarhizium anisopliae. J. Invertebr. Pathol. 105, 132-138. doi: 10.1016/j.jip.2010.05.012

Livak, K. J., and Schmittgen, T. D. (2001). Analysis of relative gene expression data using real-time quantitative PCR and the 2- $\Delta \Delta \mathrm{CT}$ method. Methods 25 , 402-408. doi: 10.1006/meth.2001.1262

Lomer, C. J., Bateman, R. P., Johnson, D. L., Langewald, J., and Thomas, M. (2001). Biological control of locusts and grasshoppers. Annu. Rev. Entomol. 46, 667-702. doi: 10.1146/annurev.ento.46.1.667

Luo, S., He, M., Cao, Y., and Xia, Y. (2013). The tetraspanin gene MaPls1 contributes to virulence by affecting germination, appressorial function and enzymes for cuticle degradation in the entomopathogenic fungus, Metarhizium acridum. Environ. Microbiol. 15, 2966-2979. doi: 10.1111/1462-2920.12166

Mienda, B. S., Idi, A., and Umar, A. (2011). Microbiological features of solid state fermentation and its applications - An overview. Res. J. Biotech. 2, 21-26.

Muñiz-Paredes, F., Miranda-Hernández, F., and Loera, O. (2017). Production of conidia by entomopathogenic fungi: from inoculants to final quality tests. World J. Microbiol. Biotechnol. 33:57. doi: 10.1007/s11274-017-2229-2

Oostra, J., le Comte, E. P., van den Heuvel, J. C., Tramper, J., and Rinzema, A. (2001). Intra-particle oxygen diffusion limitation in solid-state fermentation. Biotechnol. Bioeng. 75, 13-24. doi: 10.1002/bit.1159

Ortiz-Urquiza, A., and Keyhani, N. O. (2015). Stress response signaling and virulence: insights from entomopathogenic fungi. Curr. Genet. 61, 239-249. doi: 10.1007/s00294-014-0439-9

Peacock, A. J. (1998). ABC of oxygen: oxygen at high altitude. BMJ 1998, 10631066. doi: $10.1136 / \mathrm{bmj} .317 .7165 .1063$

Peng, G. X., Wang, Z. K., Yin, Y. P., Zeng, D. Y., and Xia, Y. X. (2008). Field trials of Metarhizium anisopliae var. acridum (Ascomycota:Hypocreales) against oriental migratory locusts, Locusta migratoria manilensis (Meyen) in Northern China. Crop Prot. 27, 1244-1250. doi: 10.1016/j.cropro.2008.03.007

Plapp, B. V., Lee, A. T., Khanna, A., and Pryor, J. M. (2013). Bradykinetic alcohol dehydrogenases make yeast fitter for growth in the presence of allyl alcohol. Chem. Biol. Interact. 202, 104-110. doi: 10.1016/j.cbi.2012.11.010

Quesada-Moraga, E., Navas-Cortes, J. A., Maranhao, E. A., Ortiz-Urquiza, A., and Santiago-Alvarez, C. (2007). Factors affecting the occurrence and distribution of entomopathogenic fungi in natural and cultivated soils. Mycol. Res. 111, 947-966. doi: 10.1016/j.mycres.2007.06.006

Rahardjo, Y. S., Sie, S., Weber, F. J., Tramper, J., and Rinzema, A. (2005). Effect of low oxygen concentrations on growth and alpha-amylase production of Aspergillus oryzae in model solid-state fermentation systems. Biomol. Eng. 21, 163-172. doi: 10.1016/j.bioeng.2005.01.001

Rangel-Porras, R. A., Meza-Carmen, V., Martinez-Cadena, G., Torres-Guzman, J. C., Gonzalez-Hernandez, G. A., Arnau, J., et al. (2005). Molecular analysis of an NAD-dependent alcohol dehydrogenase from the zygomycete Mucor circinelloides. Mol. Genet. Genomics 274, 354-363. doi: 10.1007/s00438-005$0025-4$

Reid, M. F., and Fewson, C. A. (1994). Molecular characterization of microbial alcohol dehydrogenases. Crit. Rev. Microbiol. 20, 13-56. doi: 10.3109/ 10408419409113545

Rotariu, L., Arvinte, A., Litescu, S. C., and Bala, C. (2004). Fast enzymatic method for acetaldehyde determination in wine quality control. Univ. Ann. Bucharest Chem 13, 105-110.

Stanley, G. A., Douglas, N. G., Every, E. J., Tzanatos, T., and Pamment, N. B. (1993). Inhibition and stimulation of yeast growth by acetaldehyde. Biotechnol. Lett. 15, 1199-1204. doi: 10.1007/BF00130297

Sun, B. D., and Liu, X. Z. (2008). Occurrence and diversity of insect-associated fungi in natural soils in China. Appl. Soil Ecol. 39, 100-108. doi: 10.1016/j.apsoil. 2007.12.001

Tlecuitl-Beristain, S., Viniegra-Gonzalez, G., Diaz-Godinez, G., and Loera, O. (2010). Medium selection and effect of higher oxygen concentration pulses on Metarhizium anisopliae var. lepidiotum conidial production and quality. Mycopathologia 169, 387-394. doi: 10.1007/s11046-009-9268-7 
Yubak Dhoj, G. C., Kellar, S., Nagel, P., and Kafle, L. (2008). Virulence of Metarhizium anisopliae and Beauveria bassiana against common white grubs in Nepal. Formosan Entomol. 28, 11-20.

Zhou, J., Shao, X., Olson, D. G., Murphy, S. J., Tian, L., and Lynd, L. R. (2017). Determining the roles of the three alcohol dehydrogenases (AdhA, AdhB and AdhE) in Thermoanaerobacter ethanolicus during ethanol formation. J. Ind. Microbiol. Biotechnol. 44, 745-757. doi: 10.1007/s10295-016-1896-6

Zimmermann, G. (2007). Review on safety of the entomopathogenic fungus Metarhizium anisopliae. Biocontrol. Sci. Technol. 17, 879-920. doi: 10.1080/ 09583150701593963
Conflict of Interest Statement: The authors declare that the research was conducted in the absence of any commercial or financial relationships that could be construed as a potential conflict of interest.

Copyright ( 12018 Zhang, Cao and Xia. This is an open-access article distributed under the terms of the Creative Commons Attribution License (CC BY). The use, distribution or reproduction in other forums is permitted, provided the original author(s) and the copyright owner(s) are credited and that the original publication in this journal is cited, in accordance with accepted academic practice. No use, distribution or reproduction is permitted which does not comply with these terms. 